\title{
Uruguay y la cirugía del siglo XXI. Nuevos desafíos desde viejas ideas...
}

\author{
Uruguay and the $21^{\text {st }}$ century surgery. New challenges from old ideas...
}

\author{
Luis Ruso Martínez ${ }^{1}$
}

DOI: $10.31837 /$ cir.urug/3.2.8

Recibido:

21 de febrero de 2019

Aceptado: $\quad 5$ de abril de 2019

La historia de la cirugía, prácticamente comienza hace aproximadamente 150 años. Lo previo es la oscuridad de la ignorancia; recién en 1543 la anatomía sale de los conceptos galénicos y 100 después Harvey describe la circulación de la sangre, seguramente inspirado en las ideas de Descartes. La cirugía - con más leyenda que conceptos- cabalgó entre amputaciones y suturas durante siglos, sin saber entrar con éxito terapéutico a la cavidad celomica. En suma no pasó nada hasta que entre 1850-1900 los aportes científicos de Lister y Semmelweis comenzaron la era del conocimiento de la infección quirúrgica y la antisepsia y con Claude Bernard se inicia la "medicina experimental "que rápidamente se transformaría en fisiopatología y a partir de esta ,una nueva forma de pensamiento médico , que revolucionaria los tratamientos.

A lo largo del siglo XX, una serie de avances científicos permitieron superar las grandes barreras de la cirugía: el dolor, la hemorragia y la infección. Este cambio del pensamiento médico, que culmina en las definiciones de Alma Ata con una nueva definición de salud /enfermedad; así como el exponencial desarrollo de la tecnología, le confieren al ejercicio clínico dos características: el cambio permanente, lo efímero de la verdad científica y por consecuencia la caída de los paradigmas técnicos.

${ }^{1}$ Profesor Cirugía General. Clínica Quirúrgica 3. Hospital Maciel. Facultad de Medicina, Universidad de la República. Montevideo. Uruguay. Irusomartinez@gmail.comORCID https://orcid.org/0000-0003-4206-4304 
Quienes ejercimos la cirugía en los últimos 30 años, hemos visto crecer como hongos aparatos y sistemas que han modificado en forma permanente nuestra conducta quirúrgica y han mejorado notoriamente los resultados de la cirugía. Desde los antibióticos, asociados al drenaje de abscesos, la hemostasis, la tecnología de sellado de vasos, la sutura mecánica, que junto con el perfeccionamiento de las imágenes, abrieron las puertas a los trasplantes, la cirugía de alta complejidad y los abordajes MMI; así como los sistemas de soporte sistémico, concentrados en las unidades de medicina intensiva, que habilitaron el control de daños e incrementaron el manejo del abdomen abierto y la cirugía en varios tiempos.

El derrumbe de los paradigmas fue y son permanentes. Dos ejemplos. El trasplante hepático de la mano de la segmentación de Couinaud y el control de la hemorragia y la coagulopatía, arrasó con todos los conceptos de los 100 años previos. En 1931 Caprio $^{(1)}$ hace la primera hepatectomía izquierda reglada; en los comentarios de la presentación del trabajo en la Sociedad de Cirugía del Uruguay, Caprio fue ferozmente atacado por cirujanos de la época y Mérola, su maestro, lo defendió diciendo: "tal vez algún día tengamos que operar las metástasis."(2)

Otro tanto ocurrió en cirugía digestiva, tal vez lo más ostensible sea el ejemplo de los ligamentos laterales (“alerones”) del recto. Después que Miles en 1908 propuso la amputación abdomino perineal que lleva su nombre para el cáncer de recto bajo, la “disección a dedo”, seguida de clampeos laterales y sección entre ligaduras, fue considerado un procedimiento esencial para la movilización del recto, en muchos textos quirúrgicos ${ }^{(3)}$, sin embargo, los estudios cadavéricos, no pudieron confirmar la existencia de los ligamentos laterales del recto,incluyendo autores nacionales citados en la literatura internacional sobre el tema ${ }^{(4)}$.

Así como se modificaron los paradigmas técnicos, también lo hicieron los sociales. Desde el concepto de "salvar la vida "que considera la vida como un bien individual y colectivo a preservar y sus consecuencias prácticas que podían expresar que: lo importante en la cirugía abdominal es como queda el abdomen del paciente y no lo que le pasa a la pared. ${ }^{(5)}$, estos conceptos incluyen autores de prestigio internacional, como Ivatury ${ }^{(6)}$ que dijo que la aceptación de la eventración es un hecho normal. Hoy, entrado el siglo XX, el paradigma social es salvar la vida,mantener la función y la restitución corporal ad integrum. La enfermedad se convierte en un objeto de consumo y la muerte "no existe"...o es culpa de alguien. Estos nuevos conceptos - entre otros - abonaron el terreno para la judicialización de la medicina, que tanto sufrimos.

Esta realidad, exige formar cirujanos, que a las competencias técnicas clásicas, le sumen fuertes conceptos bioéticos ,no solo para su práctica profesional sino también para planificar la investigación científica; formación en gestión clínica, contar con habilidades comunicacionales, ser capaz de trabajar en equipo y a través de redes de información y obviamente manejar un segundo idioma.

Esto impone cambios en el modelo de pensamiento quirúrgico, porque el conocimiento y la tecnología van a continuar su desarrollo en forma exponencial. El mayor desafío ya no será saber, sino saber elegir 
La diferencia la harán, no quienes posean la información, sino los tengan la mejor capacidad para tomar decisiones ,con escasa evidencia e información ilimitada. El desafío es elaborar conocimientos en base a tomas de decisiones rápidas en un mar de incertidumbre, producto de experiencias no asentadas e información no verificada.

La introducción de nuevas tecnologías y aparatos tiene un gran poder de seducción. Durante años la innovación ocurría a intervalos de tiempo que permitían evaluarlos en cuanto a su utilidad, eficacia y seguridad de uso. Este patrón se ha visto alterado tanto en los últimos años, que ha dado lugar al llamado "white wáter phenomenon" (7) porque las innovaciones, trascurren como un viaje por un rio turbulento, en el cual - apenas en forma transitoria- se transita por aguas tranquilas, que permiten pensar y evaluar. Los dilemas crecen y se hacen ingobernables y la toma de decisión está infectada de dudas e inseguridades.

Fried $^{(8)}$ un cirujano americano, propuso un esquema de reflexión basado en cuatro preguntas para orientar la toma de decisiones respecto al uso de técnicas innovadoras: a) ¿esta innovación satisface una necesidad clínica? ; b) ¿qué añade de valor a las opciones existentes?; c) ¿es financieramente sustentable?; d) ¿puede ser adoptada por la mayoría de los médicos con relativa facilidad? De este marco conceptual el principal desafío es distinguir entre lo imprescindible, lo necesario y lo superfluo adaptarse a los cambios, aprender a elegir rápidamente el mejor camino y evitar las rocas, para obtener el mejor resultado para nuestros pacientes. $^{(9)}$

Debemos repensar el dogma de la academia y reinventar los patrones tradicionales de la investigación para lograr un verdadero impacto en el campo de la salud ${ }^{(10)}$

Este modelo de formación de los jóvenes que se inician en la cirugía, incluye un fuerte desafíopara las estructuras educativas,facultades de medicina a través de planes de estudio, gobiernos y gremios para sostener la educación médica permanente y para lograr condiciones de trabajo y remuneración acorde a las exigencias de la profesión.

Estas exigencias conllevan cambios en las formas de trabajo, dentro de lo cual es imprescindible la gestión por procesos: ajustarnos a guías asistenciales, sin perder la individualidad de cada paciente, ser capaces de trabajar en equipo y redes profesionales. La responsabilidad compartida mejora la calidad, apunta a la excelencia y da seguridad a las decisiones. Las competencias en cosas concretas como cuidados del paciente, habilidades interpersonales para tener un trato afectuoso con el paciente y su familia y profesionalismo entendido éste como la unión conocimiento, responsabilidad y valores morales aplicados.

También es un desafío ético, aceptar el error y los eventos adversos,así como analizarlos en profundidad desde la perspectiva de la mejoría de la calidad y la gestión; desterrando el consagrado e inútil enfoque punitivo de los mismos. 
También hay un una nueva sociología quirúrgica .Llegaron las mujeres - con excelente resultados técnicos y alto aporte de humanismo - con su forma de vivir,sus hijos y sus familias, esto está cambiando el ejercicio de la especialidad y sobre todo va a cambiar el modelo del trabajo quirúrgico.

Otro factor de impacto es que en poco tiempo cinco generaciones diferentes - desde los babyboomers hasta los millenians - van a estar integradas en el mismo mercado académico y profesional, cada una de ellas con perspectivas y exigencias laborales, personales y educativas diferentes. El impacto ya es tremendo, nosotros con el libro y la RX de abdomen de pie, los milennians tomando decisiones desde la WEB.

Todo vale, todo aporta, pero las consecuencias deben pensarse, equilibrarse y ponerse en la perspectiva del mejor ejercicio de la cirugía.

\section{Los desafíos del futuro}

Hemos expuesto someramente la complejidad de los días que vive nuestra profesión.

El ejercicio de la cirugía tiene una base fundamental que es saber operar. Este principio que nos define no se puede sustituir por nada.

Ahora bien, la visión tradicional del entrenamiento quirúrgico basado en la adquisición de destrezas técnicas,a través de la tutorización de un cirujano experto, es por lo menos insuficiente.

El entrenamiento quirúrgico tiene claramente niveles de complejidad que se deben desarrollar en forma simultánea: a) Micro nivel. El hombre frente al desafío individual delaprendizaje; cuya base es el estudio,que integra conocimiento y permite desarrollar un espíritu crítico y tomar decisiones correctas y fundadas. La adquisición de la destreza quirúrgica, como se dijo eje fundamental de nuestra práctica clínica y gestión de economía de la salud, para las decisiones técnicas tengan sustento en la ecuación costo-beneficio. b) Meso nivel. El cirujano integrado un equipo multidisciplinario y a una organización, formando parte de un proceso de responsabilidad compartida. c) Macro-nivel. Formando parte redes de nivel regional, de país e internacionales, intercambiando información, aprendiendo y enseñando. Como parte un proceso de educación médica continua. Pero además, es imprescindible generar una cultura decontrol del rendimiento quirúrgico, en términos de resultados (morbimortalidad); eficacia, medida en tiempo y seguridad a través del análisis de errores y eventos adversos ${ }^{(11)}$

No es sostenible vivir bajo el stress permanente de la complejidad inmanejable. Debemos tratar de hacer una cirugía más simple, estandarizada y efectiva, que nos permita ofrecer una asistencia de alta calidad y económicamente sustentable.

En definitiva, creo que cada joven tiene implícita la capacidad y la responsabilidad de formar el futuro de la cirugía en el Uruguay, más allá de influencias externas que limiten o enturbien el crecimiento y desarrollo. Deben hacerlo con fórmulas sencillas: con objetivos colectivos claros (calidad, 
excelencia,seguridad, control); acciones individuales básicas (estudio, lectura, espíritu crítico ) y permanente actitud de generación de nuevos conocimientos .

\section{Bibliografía}

1) Ruso Martinez L. Caprio and Merola: Latin American contribution to the development of liver surgery.Dig. Surg2019 36(2):124-8. doi: 10.1159/000487309.

2) Praderí R., Gomez Fossati C. Estefan A. Hepatectomía dextro-mediana por cáncer secundario de hígado.Cir. Urug. 1978;48: 250-3.

3) Wag G., Gao CH., Wei D.,Wang C., Meng W. Anatomy of the lateral ligamnts of the rectum: a controversial point of view.World J Gastroenterol. 2010;16(43):5411-5.

4) Canessa C., Miegge L., Bado J., Silveri C., Labandera D. Anatomic study of lateral pelvic lymph nodes .Implications in the treatment of rectal cancer. Dis. Colon Rectum 2004;47:297.303

5) Praderi R., Bogliaccini G., Navarro T.Cierre rápido de laparotomía con tubos de polietileno. Cir. Urug.1981; 51:144-7

6) Ivatury RR, Nallathambi M, Rao PM, Rohman M, Stahl WM. Open management of the septic abdomen: therapeutic and prognostic considerations based on APACHE II. Crit Care Med. 1989;17:511-7.

7) Pellegrini C.The surgeon of the future: anchoring innovation and science with moral values. ACS Bulletin. December 2013; 98 :8-14

8 )Fried GM. The challenges of change: Presidential address to the $69^{\circ}$ anual meeting of the Central Surgical Association, Madison,Wisconsin. Narch 2012. Surgery 2012;152:509-16.

9) Ruso L. Observations of a Latin American Visitor.AZN J. Surg. 2004;74:603

10) Fernandez -Moure J.Lost in translation: the Gap in scientific advancements and clinical application.Front Bioeng. Biothechnol 4:43. DOI: 10.3389/fbioe.2016.00043

11) Tullis T, Albert B. Measuring the user experience: collecting, analyzing and opresenting usability metrics (interactives technologies). Burlington MA:Morgan Kaufmann,2008. 